\title{
TOUCHING base
}

\section{Pancreatic pathw ays}

Studies of the molecular embryology of endodermal derivatives continue apace, with two recent papers providing new insight into the genetic control of pancreatic development. Gail Deutsch and colleagues (of Brown University) have identified a bipotential population of precursor cells in the mouse embryo that can develop into either hepatic or ventral pancreatic tissue (Development 128, 871-881; 2001; see also the News \& Views on page 355 of this issue). In a second report, Anne Grapin-Botton and colleagues (Harvard University) show that the transcription factors PdxI and Atoh5 (also known as ngn3), when ectopically expressed in the embryonic chick endoderm, are sufficient to induce pancreatic budding and endocrine cell differentiation (Genes Dev. 15, 444-454; 2001). By using an in ovo electroporation procedure specifically targeting Pdxl to endodermal regions where it is not normally expressed, the authors were able to induce epithelial cell migration into the mesenchyme. These migrating cells frequently coalesced into clumps resembling pancreatic buds. Whereas these do not express markers specific to terminally differentiated endocrine cells, the electroporation of both Pdx1 and Atoh5 is sufficient to promote the formation of islets expressing glucagon and somatostatin. As Grapin-Botton et al. note, the introduction of a select group of transcription factors into embryonic or adult stem cells could provide a readily transplantable population of cells expressing endocrine hormones.

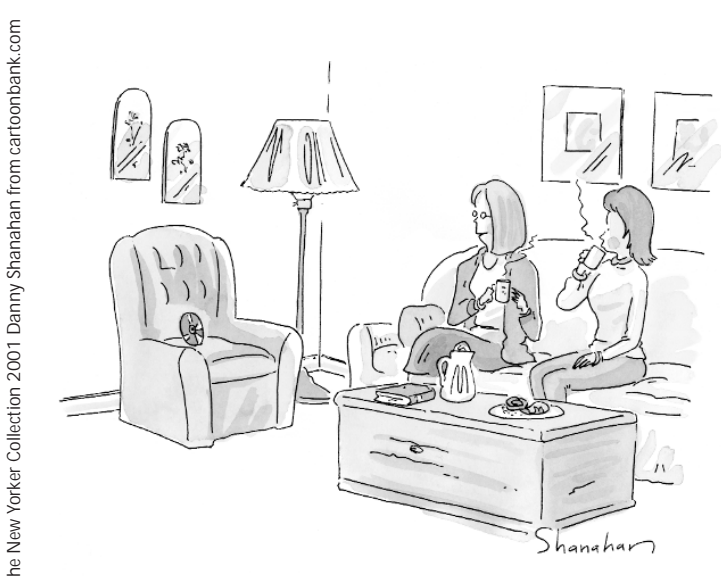

"I should have had him put into a more manageable format years ago."

\section{Blood simple}

Mad cow disease, or bovine spongiform encephalopathy, is part of a group of fatal neurodegenerative diseases called transmissible spongiform encephalopathies (TSEs). These include scrapie in sheep and Creutzfeldt-Jakob disease in humans. Humans and animals can develop TSEs after exposure to the infectious proteins, prions. As the effects of infection are observed mainly in the central nervous system (CNS), which does not lend itself to diagnostic tests, there are no reliable tests to identify infected people and animals. A study in the current issue of Nature Medicine by Gino Miele and colleagues (vol. 7, 361-364; 2001), however, indicates that a test may not be far off. They focused on the lymphoreticular system (spleen, tonsils, lymph nodes and appendix), as previous studies have shown that prions replicate in its tissues long before the effects are detected in the CNS. Using differential display, they compared the levels of expression of 10,000 transcripts from the spleens of infected mice with those of uninfected mice and identified one transcript, the levels of which are significantly decreased in the spleens of infected mice.
On omics

"Proteomics" is no longer in the lexicon of biologists alone. On 18 February 2001, William Safire discussed the word in his "On Language" column in the New York Times Magazine. Safire wrote that "proteomics" was whispered in his ear at the World Economic Forum in Davos, Switzerland-reminding him of the 1967 film The Graduate, in which Dustin Hoffman had "plastics!" uttered in his ear. This indicates the start of something big. Whereas the word is used by many, few will be aware of its origin. According to Safire, "proteomics" was coined by a graduate student, Marc Wilkins, at Macquarie University in Sydney, Australia. Wilkins, noting the evolution of "genomics" from genome, came up with "proteomics", from proteome. As he defines it, proteomics is "the study of proteins, how they're modified, when and where they're expressed, how they're involved in metabolic pathways and how they interact with one another." Safire prefers the more mellifluent "proteinomics" to "proteomics" - noting that the suffix fits snugly against an ' $n$ ': think "Nixonomics, Reaganomics and Clintonomics but not Fordomics, Carteromics or Bushomics." He is not alone in his favoring of -nomics: Beef-n-omics 4.13 is a software package developed in Australia (a coincidence, surely) to analyze strategies of managing beef cattle by integrating herd management, feed balances and economic variables. Proteomics, though, like plastics, seems to be here to stay: as we go to press, the Internet search engine Google yields approximately 32,800 entries with "proteomics" as the query, whereas a search for "proteinomics" turns up a pithy 93 hits.

\section{Drosophila goes scuba-diving}

Genetic analysis of the development of the compound eye of Drosophila has been key to unveiling molecules and signaling pathways that govern the proliferation and differentiation of human cells. Because the smallest disruption in the development of the compound eye results in the destruction of its crystalline lattice- a 'rough eye' phenotype - it has been possible to screen large numbers of mutants by visual inspection using a dissecting microscope. However, to study terminal differentiation, tissue polarity or neuronal axon guidance, which demand microscopy at higher resolution, flies must be killed and prepared for histology. In a recent issue of Development (vol. 128, 815-826; 2001), Franck Pichaud and Claude Desplan (of New York University) describe a method that allows the visualization of the fine structure of photoreceptor neurons in live flies. To overcome the refraction problems linked to the use of a high-power fluorescence microscope, they simply submerged immobilized flies in water (flies will survive such treatment for at least 20 minutes). To visualize fine neuronal organization, the authors prepared transgenic lines producing the green fluorescent protein (GFP) in specific neuronal subsets Finally, by using the FLP/FRT recombinase system - analogous to the Cre/Lox system-they could generate mosaic animals in which homozygous mutant cells were confined to patches in the eye. Use of the recombi-

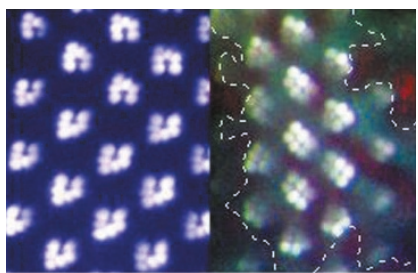
Going live. Wild-type eye (left) and a boss-mutant patch (right) in which each facet lacksone central photoreceptor cell. effect of lethal mutations in the eye, and obtain homozygous cells in one generation instead of three. Combining this with high-resoution microscopy opens the door to large-scale genetic screens for new cellular functions. Whether the combination of these techniques will usher in a period as productive as the rough-eye era remains to be seen. 